\title{
Benefit finding among parents of young children with type 1 diabetes
}

\author{
Jessica S. Pierce ${ }^{1}$ @ | Rachel Wasserman ${ }^{1}$ | Paul Enlow ${ }^{2}$ | Karen Aroian ${ }^{3}$ | \\ Joyce Lee ${ }^{4}$ | Tim Wysocki ${ }^{5}$
}

${ }^{1}$ Center for Healthcare Delivery Science, Nemours Children's Hospital, Orlando, Florida

${ }^{2}$ Center for Healthcare Delivery Science, Nemours Alfred I. duPont Hospital for Children, Wilmington, Delaware

${ }^{3}$ College of Nursing, University of Central Florida, Orlando, Florida

${ }^{4}$ Division of Pediatric Endocrinology, Child Health Evaluation and Research Unit, University of Michigan, Ann Arbor, Michigan

${ }^{5}$ Center for Healthcare Delivery Science, Nemours Children's Specialty Care,

Jacksonville, Florida

\section{Correspondence}

Jessica S. Pierce, Ph.D., Division of Behavioral Health, 13535 Nemours Parkway, \#C5741, Orlando, FL 32827.

Email: jessica.pierce@nemours.org

Funding information

National Institute of Diabetes and Digestive and Kidney Diseases, Grant/Award Number: 1DP3DK108198-03

\begin{abstract}
Benefit finding, perceived positive effects of adversity, has been associated with psychological well-being in people with chronic illnesses and with better adherence for adolescents with type 1 diabetes (T1D). Our qualitative research with parents of young children ( $<6$ years old) with T1D indicated that benefit finding (BF) is a common parental coping mechanism, but no tools exist to measure BF in parents. We determined psychometric properties of the Diabetes Benefit Finding Scale for Parents (DBFS-P), a 16-item questionnaire adapted from the validated adolescent version. Parents of young children with T1D $(n=172)$ were participants in a randomized trial of an online intervention. We examined the DBFS-P factor structure through principal component analysis (PCA); internal consistency through Cronbach's alpha; convergent validity via bivariate correlations between the DBFS-P and measures of parental depression, anxiety, T1D self-efficacy, and hypoglycemia fear; and discriminant validity via bivariate correlations between the DBFS-P and measures of parental somatization and child behavior problems. PCA revealed one factor $(56.47 \%$ variance) with Cronbach's $\alpha=0.95$. Convergent validity of the DBFS-P was supported by significant correlations with parental depression $(r=-0.35, P<0.001)$, anxiety $(r=$ $-0.20, P=0.008)$, T1D self-efficacy $(r=0.36, P<0.001)$, and hypoglycemia fear $(r=0.27, P<0.001)$. Non-significant correlations with parental somatization $(r=$ $-0.06, P=0.42)$ and child behavior problems $(r=-0.12, P=0.14)$ support its discriminant validity. The DBFS-P demonstrated good psychometric properties as a tool for assessing BF among caregivers.
\end{abstract}

KEYWORDS

benefit finding, instrument development, type 1 diabetes, young children

\section{1 | INTRODUCTION}

Type 1 diabetes (T1D) in young children ( $<6$ years old) is becoming increasingly prevalent and managing T1D in this population is challenging from medical and psychosocial perspectives. ${ }^{1,2}$ Young children with T1D (YC-T1D) have high insulin sensitivity, nocturnal hypoglycemia is common, and there is evidence indicating that the association between glycemic variability, particularly hyperglycemia, and cognitive function is more pronounced in YC-T1D than older children with T1D. ${ }^{3}$ YC-T1D also have unique developmental challenges including normative dependence upon parental caretaking ${ }^{4}$ and labile self-regulation of behavior and emotions, ${ }^{5,6}$ eating, ${ }^{7}$ 
sleep, ${ }^{8}$ and physical activity ${ }^{9}$ that may complicate T1D care. Thus, the daily management of T1D in young children is extremely complex and challenging, with the onus of these efforts falling primarily on parents.

In our conceptual model of parent and child influences on T1D management and outcomes, we postulated that the effectiveness of parental coping with the challenges related to raising a young child with T1D has both direct and indirect effects on child outcomes. ${ }^{10}$ Existing research supporting the model focuses on maladaptive coping mechanisms (eg, fear of hypoglycemia, sleep disruption), ${ }^{10}$ but positive coping strategies such as benefit finding (BF) may be especially helpful for parents of YC-T1D. BF refers to the process of perceiving positive life changes from adversity, ${ }^{11}$ and may be an important component of resiliency. ${ }^{12}$ The experience of adversity can threaten one's views of the self, but by reevaluating these views and by finding benefits, a new sense of meaning can emerge. ${ }^{13}$ Parents may therefore engage in BF as a cognitive strategy for coping with the stress related to parenting YC-T1D. ${ }^{14}$ The pervasive demands of YC-T1D care are essentially non-modifiable and so positive coping strategies that enable parents to reframe those demands could diminish parental distress substantially. Positive coping strategies such as BF may equip parents to avoid psychological fatigue and burnout that could easily be overwhelming. Indeed, higher perceptions of BF have been linked with better psychosocial functioning in parents of youth with varied physical and mental illnesses. ${ }^{15-17}$ Moreover, in adolescents with T1D, BF was related to less depressive symptoms and better self-care. ${ }^{11,18,19}$ However, no studies have quantitatively examined BF in parents of YC-T1D, in part because no validated measure of BF in parents of YC-T1D existed.

We encountered evidence of BF during our preliminary qualitative work with parents of YC-T1D in which we analyzed parents' responses to open-ended questions about parental burden, impact on parents, children, family, and other social relationships, and health care interactions. ${ }^{20}$ Content analysis revealed many spontaneous self-reports of BF among participants. The researchers organized the data into a social-ecological framework comprising five domains of influence on children's T1D outcomes. Themes about BF emerged within each domain, indicating that many parents of YCT1D perceive benefits not only for themselves, but for their children and family unit, and may use BF as a common coping mechanism. The themes derived from our qualitative work yielded the content domain for an online coping intervention for parents of YCT1D. ${ }^{20,21}$ During this preliminary work, it became clear that BF was an important psychological process to measure in the subsequent randomized controlled trial of the online coping intervention compared to usual care.

The Benefit Finding Scale (BFS) is a psychometrically sound, 17-item measure that was originally developed to assess how BF is experienced by adults living with cancer. ${ }^{23}$ The BFS has been adapted for research on BF in caregivers with other chronic and physical illnesses such as cancer, ${ }^{15,22}$ multiple sclerosis, ${ }^{23}$ and developmental disabilities. $^{24,25}$ It was also recently adapted as an self- report measure of BF in adolescents with T1D. ${ }^{19}$ In this adaptation, one item was dropped because of not being relevant to adolescents. Factor analyses performed on the original BFS, the T1D adolescent self-report version of the BFS, and several others have identified a single factor solution ${ }^{19,26-29}$ while other studies in adults with cancer $^{30,31}$ and caregivers of youth with cancer ${ }^{15}$ report the instrument as multidimensional.

In the present study, we adapted the BFS for adolescents with T1D for completion by parents of YC-T1D and determined its psychometric properties in that context. We sought to verify that this adapted measure, the Diabetes Benefit Finding Scale, Parent Version (DBFS-P), would be psychometrically sound with indications of adequate reliability and validity, including internal consistency, itemtotal correlations, bivariate correlations with measures of similar constructs (depression, anxiety, self-efficacy, and fear of hypoglycemia) as evidence of convergent validity, and non-significant bivariate correlations with measures of unrelated constructs (parental somatization and child behavior problems) as evidence of discriminant validity. Because previous research has revealed both single and multi-factor solutions for the original measure, a secondary aim was to evaluate the factor structure of this measure through exploratory factor analysis.

\section{2 | METHODS}

\section{1 | Participants and procedures}

All study procedures were approved by the site's institutional review board. Participants were 172 parents/legal caregivers of 165 YC-T1D, enrolled in a 1-year randomized controlled trial (RCT) comparing an online coping intervention plus usual care to usual care only. ${ }^{21}$ Participants were recruited via emails to eligible parents of YC-T1D seen at a multisite children's health system, diabetes-focused websites, blogs, and social media groups, or contacts with T1D clinicians in the United States. Prospective participants received an email or online announcement with basic information about the project and a link to an electronic informed consent form via Research Electronic Data Capture. Following electronic authentication and submission of informed consent, participants completed a demographic information form and study questionnaires. All data reported in this paper were collected as part of the RCT Baseline evaluation, which occurred prior to randomization to the study conditions.

Participants provided an email address and contact information to enable contact during the study and compensation. Both parents of the same child were encouraged to participate and were instructed to complete study measures separately. Participants were paid $\$ 25$ on a reloadable study debit card upon completion of study questionnaires. Study eligibility criteria included: (a) parent or legal guardian of a child diagnosed with T1D currently $<6$ years old, (b) ability to access the internet at least several times per week, (c) living in the United States during the study time period, and (d) ability to read and write in English. Cross-sectional baseline data from all participants who 
TAB LE 1 Demographic characteristics of parent participants and children with T1D

\begin{tabular}{|c|c|c|}
\hline Child characteristics & Percent (n) & Mean (SD) \\
\hline Age (years) & & 3.83 (1.28) \\
\hline Duration of T1D (years) & & 1.61 (1.17) \\
\hline Most recent HbA1c (\%) & & $7.91(1.42)$ \\
\hline Gender, \% female & $41.9(72)$ & \\
\hline \multicolumn{3}{|l|}{ Race } \\
\hline Caucasian & $80.2(138)$ & \\
\hline African American & $2.3(4)$ & \\
\hline Multiple & $7.6(13)$ & \\
\hline \multicolumn{3}{|l|}{ Ethnicity } \\
\hline Hispanic & $9.3(16)$ & \\
\hline Non-Hispanic & $84.3(145)$ & \\
\hline \multicolumn{3}{|l|}{ Insulin Regimen } \\
\hline Insulin pump & $56.4(97)$ & \\
\hline Multiple daily injections & $37.8(65)$ & \\
\hline Conventional/sliding scale & $1.7(3)$ & \\
\hline $\begin{array}{l}\text { Use of continuous glucose } \\
\text { monitor, \% yes }\end{array}$ & $82.0(141)$ & \\
\hline Participant characteristics & Percent (n) & Mean (SD) \\
\hline Age (years) & & $34.20(5.92)$ \\
\hline \multicolumn{3}{|l|}{ Relationship to child } \\
\hline Biological mother & $91.3(157)$ & \\
\hline Biological father & $7.6(13)$ & \\
\hline Stepfather & $0.6(1)$ & \\
\hline Adoptive mother & $0.6(1)$ & \\
\hline \multicolumn{3}{|l|}{ Education } \\
\hline Less than seventh grade & $0.6(1)$ & \\
\hline High school diploma & $10.5(18)$ & \\
\hline Some college/technical school & $32.6(56)$ & \\
\hline Bachelor's Degree & $33.7(58)$ & \\
\hline Graduate Degree & $22.7(39)$ & \\
\hline \multicolumn{3}{|l|}{ Occupation } \\
\hline Not employed outside home & $32.0(55)$ & \\
\hline Operational/technical level & $20.3(35)$ & \\
\hline Managerial level & $22.7(39)$ & \\
\hline Professional level & $18.0(31)$ & \\
\hline \multicolumn{3}{|l|}{ Household annual income } \\
\hline$<\$ 25 \mathrm{~K}$ & $9.9(17)$ & \\
\hline$\$ 26 \mathrm{~K}-50 \mathrm{~K}$ & $19.7(34)$ & \\
\hline$\$ 51 \mathrm{~K}-\$ 75 \mathrm{~K}$ & $16.9(29)$ & \\
\hline$\$ 76 \mathrm{~K}-\$ 100 \mathrm{~K}$ & $17.4(30)$ & \\
\hline$\$ 101 \mathrm{~K}-\$ 150 \mathrm{~K}$ & $19.8(34)$ & \\
\hline$>\$ 150 \mathrm{~K}$ & $14.6(25)$ & \\
\hline
\end{tabular}

completed the DBFS-P ( $N=172)$ were used in this report. Table 1 shows the demographic and clinical characteristics of the 172 participants and their 165 YC-T1D.

\section{2 | Measures}

\subsection{1 | Demographic and Health Information Questionnaire}

This measure collected the demographic and clinical information reported in Table 1.

\subsection{2 | Diabetes Benefit Finding Scale for Parents}

The 16-item Diabetes Benefit Finding Scale for Parents (DBFS-P) was adapted for this study from the adolescent self-report version of the BFS. $^{26}$ The content of the 16 items remained identical, but the item stem was changed from "Having diabetes..." to "Raising a young child with type 1 diabetes..." The DBFS-P assesses how BF is experienced by parents of YC-T1D (eg, "Raising a young child with type 1 diabetes has led me to be more accepting of things."). Participants rate the extent to which each item applied to them, using a five-point Likert scale ranging from 1 (not at all) to 5 (extremely). The total score of the scale can range from 16 to 80 , where higher values indicate a higher degree of benefit finding.

\subsection{3 | Brief Symptom Inventory-18}

Participants reported on their own general psychological distress using the Brief Symptom Inventory-18 (BSI-18), a widely used self-report screening measure that yields standardized scores for Somatization, Depression, Anxiety and a Global Severity Index. ${ }^{32}$ Respondents rated their current status in terms of 18 physical and emotional complaints from 0 (not at all) through 4 (very much). Construct validity has been demonstrated through high positive correlations between the BSI-18 subscales and Global Severity Index and the corresponding scales of its longer progenitor, the symptom checklist-90-revised. ${ }^{32}$ In the present study, the BSI-18 demonstrated strong internal consistency for the subscales ( $\alpha$ 's $=0.76-0.85)$ and Global Severity Index ( $\alpha=0.91)$.

\subsection{4 | Parental Self-Efficacy Scale for Diabetes Management}

The 8-item Parental Self-Efficacy Scale for Diabetes Management $\left(\right.$ PSESDM) ${ }^{33}$ was adapted from a prior measure for adult patients to measure parental self-efficacy for diabetes management of young children. Parents rate their agreement with each item from 1 (strongly disagree) to 5 (strongly agree). A higher score on the PSESDM represents greater parental confidence in managing their child's diabetes. Criterion validity was demonstrated through associations between higher scores on the PSESDM and more favorable HbA1C and child quality of life. ${ }^{33}$ In the present study, the PSESDM demonstrated good internal consistency ( $\alpha=0.82$ ). 


\subsection{5 | Hypoglycemic Fear Survey-Parents of Young Children}

Parental fear of their child's hypoglycemia was assessed with the 26-item Hypoglycemic Fear Survey-Parents of Young Children (HFSPYC). ${ }^{34}$ It yields two subscales, assessing parents' behaviors related to preventing hypoglycemia (10 items) and their worry about their children's hypoglycemia (16 items). Parents rate how often each item is true for them from 1 (never) to 5 (very often). Higher scores indicate greater fear of hypoglycemia. The HFS-PYC demonstrated good testretest reliability for the Total $(r=0.91, P<0.001)$, Behavior $(r=0.73$, $P<0.001)$, and Worry $(r=0.91, P<0.001)$ scales. Higher levels of fear of hypoglycemia were associated with higher mean daily blood glucose levels. ${ }^{34}$ In the present study, the HFS-PYC demonstrated adequate internal consistency on the Total $(\alpha=0.92)$, Behavior ( $\alpha=0.68)$, and Worry $(\alpha=0.94)$ scales.

\subsubsection{Eyberg Childhood Behavior Inventory}

The Eyberg Childhood Behavior Inventory (ECBI) is a widely used parent-report measure of problematic behaviors of children $\geq 2$ years old. ${ }^{35}$ In the current study, eight participants with children $<2$ years old were not included in analyses involving the ECBI. Parents rate the frequency of 36 child behaviors on a Likert scale, that ranges from 1 (never) to 7 (always) (Intensity scale), and to then indicate whether they consider each behavior to be a problem (yes/no) (Problem scale). Higher scores on the Intensity scale indicate more frequent potentially problematic child behaviors and higher scores on the Problem scale indicate greater perception that those behaviors are problematic The $\mathrm{ECBI}$ Intensity scale has also demonstrated evidence of construct validity in a sample of parents of young children (ages 3 to 5 years) through significant correlations with observational measures of child negative affect, non-acceptance, and dominance (convergent validity) and non-significant correlations with observational measures of child positive affect or submissiveness (discriminant validity). ${ }^{35}$ In the present study, the ECBI demonstrated excellent internal consistency for the Intensity scale $(\alpha=0.93)$ and the Problem scale $(\alpha=0.91)$.

\section{3 | Data analysis}

All statistical analyses were performed using SPSS Statistics Version 25 (IBM, Inc, Chicago, IL). To determine the DBFS-P factor structure, the investigators performed exploratory principal components analysis. Then, the investigators sought to identify any covariates among the various demographic/clinical variables that were measured for which statistical adjustments should be applied to DBFS results. Correlations (for continuous variables), $t$ tests (for dichotomous variables), and ANOVAS (for categorical variables) were examined between the DBFS-P and demographic/clinical variables to determine if any were related significantly to DBFS-P scores. Alpha was set at 0.05 for all tests. Internal consistency of the DBFS-P was evaluated using Cronbach's alpha coefficient. Convergent validity of the DFBS-P was evaluated by calculating Pearson $r$ correlations between the DFBS-P and the BSI-18 Depression and Anxiety Subscales, PSESDM, and HFS-PYC. Discriminant validity was assessed by calculating Pearson $r$ correlations between DBFS-P scores and those obtained on the BSI Somatization Subscale and ECBI measures, because those measures were not expected to correlate significantly with DBFS-P scores.

\section{3 | RESULTS}

\section{1 | Descriptive statistics}

Table 1 shows demographic characteristics of the 172 parent participants and their 165 YC-T1D. The parents were predominantly Caucasian (86.3\%), Non-Hispanic (90.1\%), mothers (91.3\%), and the sample reflected above average educational and occupational attainment, household income, and frequency of general and T1D-specific Internet use. Mean (SD) age of their children was 3.83 (1.28) years. Many were using insulin pumps (56.4\%) and/or continuous glucose monitoring (82.0\%). Parents reported an average most recent $\mathrm{HbA} 1 \mathrm{c}$ of $7.9 \%(\mathrm{SD}=1.4 \%)$.

Of the possible score range on the DBFS-P of $16-80$, the mean score in this sample was 52.59 ( $S D=14.83$; range 19-80). On all 16 items, respondents endorsed the full range of responses (ie, 1 to 4). Item means ranged from $2.37(\mathrm{SD}=1.43)$ to $3.67(\mathrm{SD}=1.08)$. Item skewness ranged from -0.60 to $0.59(\mathrm{SE}=0.19)$ and kurtosis ranged from -1.13 to -0.60 (SE $=0.37$ ); total score skewness $=-0.08$ and kurtosis $=-0.77$, indicating normal skewness, but a negative kurtosis (ie, distribution is relatively thin in the tails) for most items and the DBFS-P total score. See Table 2 for the frequencies of responses to each item.

The DBFS-P total score was not significantly correlated with child age, T1D duration, HbA1C, or socioeconomic status computed using Hollingshead's (1975) four-factor index (Table 3), and did not differ significantly across child gender, ethnicity, race, health insurance coverage, insulin regimen, use of continuous glucose monitoring, parental relationship to child with $\mathrm{T} 1 \mathrm{D}$, highest parental education, or household annual income.

\section{2 | Factor structure}

To examine the component structure of the DBFS-P, exploratory principal components analysis was conducted. The Kaiser-Meyer-Olkin measure of sampling adequacy was satisfactory, and the Bartlett's Test of Sphericity reached statistical significance $(P<0.001)$, indicating that principal components analysis was acceptable. Three components had eigenvalues $>1$, but the scree plot suggested that only one factor lie above the elbow. ${ }^{36}$ Therefore, item loadings were examined for both one- and three-component solutions.

Principal components analysis using direct oblimin (oblique) rotation revealed that the three component scale accounted for $70.48 \%$ of the variance across all 16 items. All items loaded $>0.40$ on at least one component. However, there were statistical and conceptual problems with the resultant patterns. Factor 1 included seven items that loaded $>0.38$, but items cross-loaded between factors. Specifically, one of the seven items also loaded $>0.35$ on Factor 2 and one item 
TAB LE 2 Items, response options, component matrix, and item-total correlations for the Diabetes Benefit Finding Scale for Parents

Instructions: Raising a young child with type 1 diabetes has...

\begin{tabular}{|c|c|c|c|c|c|c|c|c|}
\hline & $\begin{array}{l}\text { Not at all } \\
\text { (\%) }\end{array}$ & $\begin{array}{l}\text { A little } \\
(\%)\end{array}$ & $\begin{array}{l}\text { Moderately } \\
\text { (\%) }\end{array}$ & $\begin{array}{l}\text { Quite a bit } \\
\text { (\%) }\end{array}$ & $\begin{array}{l}\text { Extremely } \\
\text { (\%) }\end{array}$ & Mean \pm SD & $\begin{array}{l}\text { Component } \\
\text { matrix }\end{array}$ & $\begin{array}{l}\text { Item-total } \\
\text { correlation }\end{array}$ \\
\hline 1. Led me to be more accepting of things & 5.8 & 18.6 & 31.4 & 23.8 & 20.6 & $3.34 \pm 1.17$ & 0.80 & 0.75 \\
\hline $\begin{array}{l}\text { 2. Taught me how to adjust to things } \\
\text { I cannot change }\end{array}$ & 1.2 & 15.7 & 25.0 & 30.8 & 27.3 & $3.67 \pm 1.08$ & 0.81 & 0.75 \\
\hline 3. Helped me take things as they come & 1.2 & 14.5 & 29.1 & 32.6 & 22.7 & $3.61 \pm 1.03$ & 0.79 & 0.74 \\
\hline 4. Brought my family closer together & 8.7 & 24.4 & 22.1 & 24.4 & 20.3 & $3.23 \pm 1.27$ & 0.75 & 0.71 \\
\hline 5. Made me more sensitive to family issues & 7.0 & 17.4 & 29.1 & 24.4 & 22.1 & $3.37 \pm 1.21$ & 0.77 & 0.72 \\
\hline 6. Taught me that everyone has a purpose in life & 13.4 & 19.2 & 22.7 & 23.8 & 20.9 & $3.20 \pm 1.33$ & 0.79 & 0.76 \\
\hline 7. Shown me that all people need to be loved & 9.9 & 11.6 & 23.8 & 26.2 & 28.5 & $3.52 \pm 1.28$ & 0.75 & 0.71 \\
\hline $\begin{array}{l}\text { 8. Helped me become more focused on priorities, } \\
\text { with a deeper sense of purpose in life }\end{array}$ & 5.8 & 14.0 & 21.5 & 29.7 & 29.1 & $3.62 \pm 1.21$ & 0.79 & 0.75 \\
\hline $\begin{array}{l}\text { 9. Made me more aware and concerned for } \\
\text { the future of all human beings }\end{array}$ & 9.3 & 18.0 & 23.3 & 24.4 & 25.0 & $3.38 \pm 1.29$ & 0.74 & 0.71 \\
\hline 10. Taught me to be patient & 7.0 & 16.9 & 23.3 & 28.5 & 24.4 & $3.47 \pm 1.23$ & 0.74 & 0.69 \\
\hline 11. Led me to deal better with stress and problems & 19.8 & 24.4 & 30.2 & 15.1 & 10.5 & $2.72 \pm 1.24$ & 0.73 & 0.69 \\
\hline $\begin{array}{l}\text { 12. Led me to meet people who have become some } \\
\text { of my best friends }\end{array}$ & 41.3 & 17.4 & 16.3 & 13.4 & 11.6 & $2.37 \pm 1.43$ & 0.52 & 0.49 \\
\hline $\begin{array}{l}\text { 13. Contributed to my overall emotional and } \\
\text { spiritual growth }\end{array}$ & 22.1 & 23.3 & 25.0 & 15.7 & 14.0 & $2.76 \pm 1.33$ & 0.73 & 0.70 \\
\hline $\begin{array}{l}\text { 14. Helped me become more aware of the love and } \\
\text { support available from other people }\end{array}$ & 12.2 & 20.9 & 23.8 & 20.3 & 22.7 & $3.20 \pm 1.33$ & 0.76 & 0.72 \\
\hline 15. Helped me realize who my real friends are & 12.8 & 11.0 & 16.9 & 30.2 & 29.1 & $3.52 \pm 1.35$ & 0.69 & 0.65 \\
\hline $\begin{array}{l}\text { 16. Helped me become a stronger person, more able } \\
\text { to cope effectively with future life challenges }\end{array}$ & 5.2 & 11.6 & 27.9 & 27.3 & 27.9 & $3.61 \pm 1.16$ & 0.83 & 0.79 \\
\hline
\end{tabular}

loaded $>0.38$ on Factor 3. Factor 2 included four items all loading $>0.46$, two of which loaded $>0.34$ on Factor 1 . Factor 3 included five items that loaded $>0.40$, one of which also loaded $>0.33$ on Factor 1. No clear pattern arose in terms of conceptual or theoretical grouping of items falling into each factor. For example, Factor 2 included two items about friends (Items 12 and 15), but also included an item about emotional and spiritual growth (Item 13) which was similar to an item on Factor 3 about sense of purpose (Item 8). Another example is that Factor 1 included an item about taking things as they come (Item 3), whereas Factor 3 included a similar item about being more patient (Item 10).

For the one-component solution, principal components analysis revealed that the single component accounted for $56.47 \%$ of the variance across all 16 items. All items had factor loadings and communalities $>0.52$. Given the potential statistical and theoretical issues with the three-component solution and the fact that uni-dimensionality is more parsimonious, the one-component solution was selected for further analyses. All 16 items were retained on the DFBS-P. Table 2 shows the component matrix for the one-factor solution.

\subsection{Reliability and validity}

Cronbach's alpha for the DBFS-P total score indicated good internal consistency, $\alpha=0.95$. Corrected item-total correlations ranged from 0.49 to
0.79 and Cronbach's alpha did not improve by deleting any items. Interitem correlations ranged from 0.20 to 0.84 . Thus, all items were retained in the final version of the DBFS-P. The DBFS-P demonstrated convergent validity with parental BSI-18 scores for Depression $(r=-0.35$, $P<0.001)$ and Anxiety $(r=-0.20, P=0.008)$ and the PSESDM $(r=0.36$, $P<0.001$ ) (Table 3). Evidence supporting the discriminant validity consisted of non-significant correlations with the BSI-18 Somatization Subscale $(r=-0.06, P=0.42)$, ECBI Intensity scale $(r=-0.12, P=0.14)$, and ECBI Problem scale $(r=0.02, P=0.82$ ) (Table 3 ).

Somewhat surprisingly, the DBFS-P was significantly correlated with the HFS-PYC total scale $(r=0.27, P<0.001)$ in a positive, rather than negative, direction. Thus, a higher level of BF was moderately associated with greater parental fear of hypoglycemia. In examining the distribution of scores on the HFS-PYC, parents obtained a mean of 76.40 (SD = 18.86) with a possible range of 26 to 130 and an actual range of 37 to 117 . Figure 1 shows the scatterplot of the correlation between the DBFS-P and HFS-PYC.

\section{4 | DISCUSSION}

This study explored the psychometric properties and performance of the 16-item DFBS-P in 172 parents of YC-T1D. The adapted measure demonstrated satisfactory psychometric properties for use in this 
TAB LE 3 Correlation matrix of associations among DBFS-P total score, demographic and clinical characteristics, and measures of convergent and divergent validity

\begin{tabular}{|c|c|c|c|c|c|c|c|c|c|c|c|}
\hline & 1 & 2 & 3 & 4 & 5 & 6 & 7 & 8 & 9 & 10 & 11 \\
\hline 1. DBFS-P & & & & & & & & & & & \\
\hline 2. Child's age & 0.07 & & & & & & & & & & \\
\hline 4. $\mathrm{HbA} 1 \mathrm{c}$ & 0.08 & $-0.22 *$ & $-0.26 * *$ & & & & & & & & \\
\hline 5. SES & -0.09 & -0.06 & -0.06 & $-0.21 *$ & & & & & & & \\
\hline 7. $\mathrm{BSI}-18 \mathrm{~A}$ & $-0.20 *$ & -0.10 & -0.05 & 0.11 & -0.08 & $0.74 * *$ & & & & & \\
\hline 8. $\mathrm{BSI}-18 \mathrm{~S}$ & -0.06 & -0.08 & 0.03 & $0.20 *$ & $-0.18 *$ & $0.52 * *$ & $0.64 * *$ & & & & \\
\hline 9. PSESDM & $0.36 * *$ & 0.09 & 0.07 & -0.13 & -0.04 & $-0.35 * *$ & $-0.33 * *$ & -0.11 & & & \\
\hline 10. HFS-PYC & $0.27 * *$ & -0.04 & -0.09 & 0.12 & $-0.17 *$ & 0.14 & $0.27 * *$ & $0.21 * *$ & -0.10 & & \\
\hline 11. ECBI Intensity & -0.12 & 0.06 & 0.10 & -0.11 & 0.06 & $0.20 *$ & $0.17 *$ & 0.08 & $-0.20 * *$ & 0.06 & \\
\hline
\end{tabular}

Abbreviations: BSI-18, Brief Symptom Inventory-18; DBFS-P, Diabetes Benefit Finding Scale for Parents; ECBI, Eyberg Childhood Behavior Inventory; HFS-PYC, Hypoglycemic Fear Survey-Parents of Young Children; PSESDM, Parental Self-Efficacy Scale for Diabetes Management; SES, socioeconomic status.

$* * P<0.001 ; * P<0.01$.

population. The findings highlighted a high internal consistency for the 16-item DBFS-P, which is consistent with studies using the instrument in adolescents with T1D and in adults and caregivers of children with a variety of chronic illnesses. ${ }^{15,17,19,23,27,30,31}$ These consistent findings of satisfactory internal consistency suggest that the instrument captures BF reliably in diverse adult, adolescent, and caregiver populations.

The results from this study also indicated that the DBFS-P measures a unidimensional construct, which is consistent with findings reported by the original instrument authors, ${ }^{26}$ studies of adolescents with $\mathrm{T} 1 \mathrm{D},{ }^{19}$ and several other chronic illness populations. ${ }^{27-29}$ The principal component analysis in this study identified all factors loading onto one component at 0.50 and above, explaining over $50 \%$ of the variance. Both of these features provide evidence for a solid one-factor solution for the DFBS-P. Although three studies found a multidimensional structure for the $\mathrm{BFS}^{15,30,31}$ and $\mathrm{BF}$ can theoretically be understood in terms of multiple factors, there is no presumption that the underlying component constructs are distinct, orthogonal, or differentially predictive. Results of the current study indicate that the use of a single factor for the DBFS-P in parents of YC-T1D is theoretically and statistically sound for measurement of $\mathrm{BF}$ in this population.

Convergent validity of the DBFS-P was supported by significant relations between diabetes-specific BF and all predicted constructs.
FIGURE 1 Scatterplot of the Diabetes Benefit Finding Scale for Parents (DBFSP) and the Hypoglycemia Fear SurveyParents of Young Children (HFS-PYC)

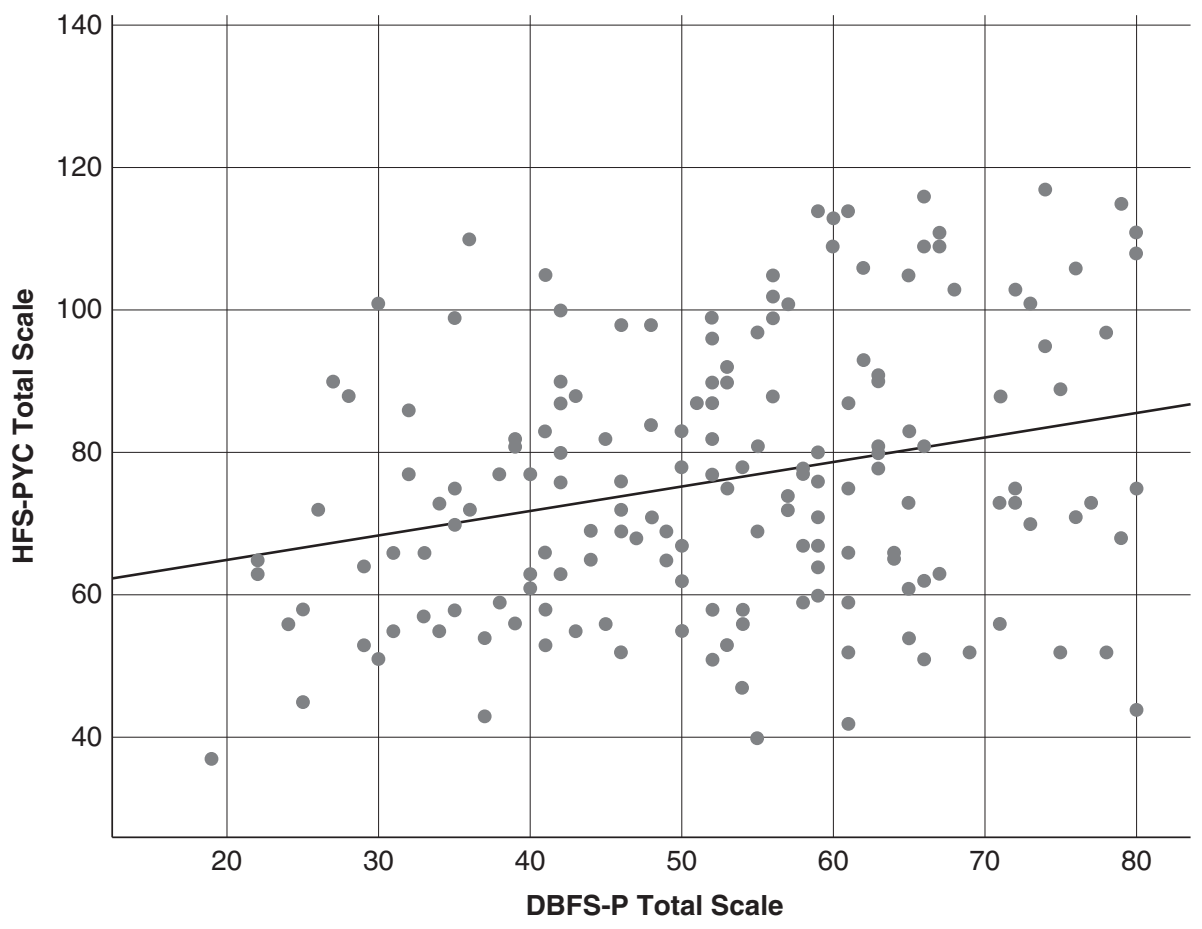


First, significant negative relations were found between diabetesspecific BF and both depression and anxiety. This is consistent with the cognitive model of depression and anxiety in which negative automatic thoughts are formed around oneself, one's environment, and one's future making it more difficult to find benefits. ${ }^{37}$ Second, a significant positive correlation was found between diabetes-specific BF and diabetes-specific self-efficacy. Parents who are more confident in their ability to manage their child's T1D may be more inclined to find benefit in the day to day nuances associated with these tasks. The DBFS-P also demonstrated discriminant validity through nonsignificant relations with somatization and child behavior problems. There were no a priori theoretical reason to expect a strong relation between either of these variables and BF. Taken together, these findings offer strong support for construct validity of the DBFS-P.

Although we expected an inverse correlation between BF and fear of hypoglycemia, the mildly positive correlation between the two variables may also support the convergent validity of the DBFS-P. We initially reasoned that higher levels of hypoglycemia fear may lead to poorer coping strategies (eg, administering lower doses of insulin or over-treating episodes of hypoglycemia) ${ }^{34}$ making it more difficult to engage in $\mathrm{BF}$, a positive coping strategy. However, some level of fear of hypoglycemia is adaptive and could equip parents with the vigilance and attentiveness needed to protect their children from hypoglycemic episodes. ${ }^{34}$ Further investigation of the distribution of HFSPYC scores and the scatterplot of the HFS-PYC and DBFS-P (Figure 1) indicates that our sample endorsed a moderate level of fear of hypoglycemia, with few scores in a range that might be debilitating. Within this range of HFS-PYC scores, our data suggest that a moderate level of hypoglycemia fear was adaptive because it was associated with more favorable DBFS-P scores.

\section{1 | Future research}

Following from this psychometric validation of the DFBS-P, other key questions about BF can now be addressed in longitudinal studies, such as

- How does BF among parents of YC-T1D emerge and develop over time?

Findings from our cross sectional data revealed that BF was not related to duration of T1D, indicating that parents whose YC-T1D were recently diagnosed did not experience more or less BF compared to those whose young children had T1D for longer durations. In an examination of trajectories of BF in adolescents with T1D over 1.5 years, BF decreased slightly. ${ }^{11}$ The authors postulated that increasing responsibility for adolescent T1D management over time may result in a stronger awareness of the impact that T1D has on daily life, potentially undermining adolescents' ability to find benefits. ${ }^{11}$ Because BF typically emerges out of negative life stressors and may be used as a coping mechanism for relieving the stress associated with managing T1D, ${ }^{38}$ parents of YC-T1D may instead show an increase in BF over time. Longitudinal studies can clarify whether BF trajectories in parents of YC-T1D mirror or differ from those of adolescents and also reveal which parents continue to derive benefits over time.

- Does BF confer protective effects against the development of parental depression and diabetes distress?

Our cross-sectional findings revealed that parents of YC-T1D who endorsed higher levels of BF also endorsed lower levels of depression. Several authors have hypothesized that BF emerges in response to distress but, once developed, serves as a buffer against distress. ${ }^{18,39}$ Indeed, findings have been mixed in terms of relations between BF and depression/distress. Longitudinal studies of parents of YC-T1D may disentangle the dynamic relationship between BF and distress.

- Can interventions directly target and promote BF among parents of YC-T1D?

To our knowledge, there are no interventions specifically targeting BF for parents of children with T1D. Cheng and colleagues (2014) developed a cognitive behavioral BF intervention for caregivers (ie, spouse or child) of people with Alzheimer's disease, which combines psychoeducation (information and problem solving) with positive reappraisal coping that is intended to help caregivers interpret the demands of caregiving in more positive ways. ${ }^{40}$ In this pilot study, participants randomized to the BF intervention had lower levels of depression posttest when compared to those randomized to psychoeducation only, ${ }^{40}$ indicating that $\mathrm{BF}$ interventions may be effective and are feasible. Future research should focus on the development and implementation of strengths-based interventions for parents of YC-T1D, as well as the best time point(s) for such an intervention (eg, within the first year of diagnosis vs later on). The DBFS-P may be useful in identifying and monitoring specific targets for such strengthsbased interventions.

\section{2 | Limitations}

Although the psychometric properties of the DBFS-P appear promising, this study should be considered in the context of several limitations. The participants had limited racial/ethnic diversity, lower-thanaverage glycemic control, ${ }^{41}$ high use of insulin pumps and continuous glucose monitors, and comprised a convenience sample of over $90 \%$ mothers, which limits the extent to which the findings can be generalized to the entire population of parents of YC-T1D. Future research studies should focus on examination of the psychometric properties of the DBFS-P in a more demographically representative sample in order to address its limited generalizability. Despite the limited socioeconomic diversity, we achieved a broad geographic representation of parents from across the United States. Correlation analysis revealed that DBFS-P scores were largely unrelated to participants' demographic characteristics, implying that the limited diversity of the present sample may have had little effect on the reported psychometric analyses. The cross-sectional nature of the baseline trial data 
precluded analysis of the DBFS-P's sensitivity to change, test-retest reliability or longitudinal associations with potential moderators of mediators of intervention effects. Ultimately, the intervention trial's outcome data will facilitate evaluation of whether the measure is sensitive to change related to utilization of an online coping intervention designed by and for parents of young children with T1D. Evaluation of test-retest reliability may require a longitudinal observational study with administrations of the DBFS-P repeated at relatively brief intervals. As with all self-report measures, responses on questionnaires may have been impacted by response bias. Finally, the factor structure and psychometric properties were not confirmed in a second independent sample. Replication of these analyses in another sample will enhance the robustness of the findings and inform conclusions about generalizability and utility of this measure. To our knowledge, the DBFS-P has not been validated in parents of school-aged or older children with T1D, so it should only be used in parents of children less than age 6 years. Future research should focus on extending the age range of the target population.

In sum, this study showed that the DBFS-P has promising psychometric properties. The DBFS-P facilitates the examination of BF in a new population, caregivers of YC-T1D, rather than patients themselves. Future research should focus on evaluation of current BF and implementation of strengths-based interventions in parents of young children with T1D. The DBFS-P may be useful in identifying and monitoring specific targets for such strengths based interventions. Further, our ongoing randomized controlled trial will examine BF over time and evaluate whether parental BF mediates or moderates changes in other outcomes of health and well-being among YC-T1D and their parents.

\section{ACKNOWLEDGEMENTS}

This work was supported by NIH/NIDDK Grant \# DP3-DK-108198 (Drs. Wysocki and Pierce as Multiple PIs).

\section{CONFLICT OF INTEREST}

Dr. Joyce Lee is a consultant to the Type 1 Diabetes Exchange. The other authors do not have any potential sources of conflicts of interest to disclose.

\section{ORCID}

Jessica S. Pierce (D) https://orcid.org/0000-0001-6352-9225

\section{REFERENCES}

1. Pierce JS, Kozikowski C, Lee JM, Wysocki T. Type 1 diabetes in very young children: a model of parent and child influences on management and outcomes. Pediatr Diabetes. 2015;18(6):17-25. https://doi. org/10.1111/pedi.12351.

2. Patterson CC, Harjutsalo V, Rosenbauer J, et al. Trends and cyclical variation in the incidence of childhood type 1 diabetes in 26 European centres in the 25 year period 1989-2013: a multicentre prospective registration study. Diabetologia. 2018;62:408-417. https://doi.org/10. 1007/s00125-018-4763-3.

3. Barnea-Goraly N, Raman M, Mazaika P, et al., for the Diabetes Research in Children Network (DirecNet). Alterations in white matter structure in young children with type 1 diabetes. Diabetes Care. 2014; 37(2):332-340. https://doi.org/10.2337/dc13-1388.

4. Monaghan M, Helgeson V, Wiebe D. Type 1 diabetes in young adulthood. Curr Diabetes Rev. 2015;11:239-250. https://doi.org/10.1161/ STROKEAHA.113.002813.Oxidative.

5. Cole PM, Dennis TA, Smith-Simon KE, Cohen LH. Preschoolers' emotion regulation strategy understanding: relations with emotion socialization and child self-regulation. Soc Dev. 2009;18(2):324-352. https://doi.org/10.1111/j.1467-9507.2008.00503.x.

6. Wasserman RM, Hilliard ME, Schwartz DD, Anderson BJ. practical strategies to enhance executive functioning and strengthen diabetes management across the lifespan. Curr Diab Rep. 2015;15:52. https:// doi.org/10.1007/s11892-015-0622-5.

7. Cathey M, Gaylord N. Picky eating: a toddler's continuing approach to mealtime. Pediatr Nurs. 2004;30(2):101-107. http://www.ncbi.nlm. nih.gov/pubmed/15185731.

8. National Sleep Foundation. How much sleep do we really need? Sleep Res. 2016;325(5942):825-826. https://doi.org/10.1126/ science.1178713.

9. Timmons BW, Naylor PJ, Pfeiffer KA. Physical activity for preschool children-how much and how? Can J Public Health. 2007;98(Suppl 2): S122-S134. https://doi.org/10.1139/H07-112.

10. Pierce JS, Kozikowski C, Lee JM, Wysocki T. Type 1 diabetes in very young children: a model of parent and child influences on management and outcomes. Pediatr Diabetes. 2017;18(1):17-25. https://doi. org/10.1111/pedi.12351.

11. Rassart J, Luyckx K, Berg CA, Oris L, Wiebe DJ. Longitudinal trajectories of benefit finding in adolescents with type 1 diabetes. Health Psychol. 2017;36(10):977-986. https://doi.org/10.1037/hea0000 513.

12. Hilliard ME, Harris MA, Weissberg-Benchell J. Diabetes resilience: a model of risk and protection in type 1 diabetes. Curr Diab Rep. 2012; 12(6):739-748.

13. Tedeschi RG, Calhoun LG. Posttraumatic growth: conceptual foundations and empirical evidence. Psychol Inq. 2004;15(1):1-18. https:// doi.org/10.1207/s15327965pli1501_01.

14. Brand C, Barry L, Gallagher S. Social support mediates the association between benefit finding and quality of life in caregivers. J Health Psychol. 2014;21(6):1126-1136. https://doi.org/10.1177/13591053 14547244.

15. Kim Y, Schulz R, Carver CS. Benefit finding in the cancer caregiving experience. Psychosom Med. 2007;69(3):283-291. https://doi.org/10. 1097/PSY.0b013e3180417cf4.

16. Lim KK, Chong WH. Moderating effect of child's autism spectrum disorder (ASD) diagnosis on benefit finding and negative affect of parents. Am J Orthopsychiatry. 2017;87(3):357-364. https://doi.org/10. 1037/ort0000251.

17. Magliano L, Patalano M, Sagliocchi A, et al. "I have got something positive out of this situation": psychological benefits of caregiving in relatives of young people with muscular dystrophy. J Neurol. 2014;261: 188-195. https://doi.org/10.1007/s00415-013-7176-8.

18. Helgeson VS, Lopez L, Mennella C. Benefit finding among children and adolescents with diabetes. In: Park CL, Lechner SC, Antoni MH, Stanton AL. eds. Medical illness and positive life change: Can crisis lead to personal transformation? Washington, DC: American Psychological Association; 2009:65-86.

19. Tran V, Wiebe DJ, Fortenberry KT, Butler JM, Berg CA. Benefit finding, affective reactions to diabetes stress, and diabetes management among early adolescents. Health Psychol. 2011;30:212-219. https:// doi.org/10.1037/a0022378. 
20. Pierce JS, Aroian K, Caldwell C, et al. The ups and downs of parenting young children with type 1 diabetes: a crowdsourcing study. J Pediatr Psychol. 2017;42(8):846-860. https://doi.org/10.1093/jpepsy/jsx 056.

21. Wysocki T, Pierce J, Caldwell C, et al. Web-based coping intervention by and for parents of very young children with type 1 diabetes: usercentered design. JMIR Diabetes. 2018;3(4):e16. https://doi.org/10. 2196/diabetes.9926.

22. Cassidy T. Benefit finding through caring: the cancer caregiver experience. Psychol Health. 2013;28:250-266. https://doi.org/10.1080/ 08870446.2012.717623.

23. Pakenham KI. Benefit finding in multiple sclerosis and associations with positive and negative outcomes. Health Psychol. 2005;24:123132. https://doi.org/10.1037/0278-6133.24.2.123.

24. Perantie DC, Wu J, Koller JM, et al. Perception of difficulty and glucose control: effects on academic performance in youth with type I diabetes. Diabetes Care. 2007;32(3):527-533. https://doi.org/10. 1093/jpepsy/jsn024.

25. Slattery É, McMahon J, Gallagher S. Optimism and benefit finding in parents of children with developmental disabilities: the role of positive reappraisal and social support. Res Dev Disabil. 2017;65:12-22. https://doi.org/10.1016/j.ridd.2017.04.006.

26. Antoni $\mathrm{MH}$, Lehman JM, Kilbourn KM, et al. Cognitive-behavioral stress management intervention decreases the prevalence of depression and enhances benefit finding among women under treatment for early-stage breast cancer. Health Psychol. 2001;20:458-459. https:// doi.org/10.1037/0278-6133.20.6.458a.

27. Urcuyo KR, Boyers AE, Carver CS, Antoni MH. Finding benefit in breast cancer: relations with personality, coping, and concurrent wellbeing. Psychol Health. 2005;20:175-192. https://doi.org/10.1080/ 08870440512331317634.

28. Kangas M, Williams JR, Smee RI. Benefit finding in adults treated for benign meningioma brain tumours: relations with psychosocial wellbeing. Brain Impair. 2011;12(2):105-116. https://doi.org/10. 1375/BRIM.12.2.105.

29. Pascoe L, Edvardsson D. Psychometric properties and performance of the 17-item Benefit Finding Scale (BFS) in an outpatient population of men with prostate cancer. Eur J Oncol Nurs. 2015;19:169-173. https://doi.org/10.1016/j.ejon.2014.09.004.

30. Luszczynska A, Mohamed NE, Schwarzer R. Self-efficacy and social support predict benefit finding 12 months after cancer surgery: the mediating role of coping strategies. Psychol Health Med. 2005;10: 365-375. https://doi.org/10.1080/13548500500093738.

31. Llewellyn CD, Horney DJ, McGurk M, et al. Assessing the psychological predictors of benefit finding in patients with head and neck cancer. Psychooncology. 2013;22:97-105. https://doi.org/10.1002/ pon. 2065.

32. Derogatis LR, Fitzpatrick M. The SCL-90-R, the Brief Symptom Inventory (BSI), and the BSI-18. The Use of Psychological Testing for Treatment Planning and Outcomes Assessment: Vol 3: Instruments for Adults. Mahwah, New Jersey: Lawrence Erlbaum Associates; 2004. https:// doi.org/10.1037/024099.

33. Marchante AN, Pulgaron ER, Daigre A, et al. Measurement of parental self-efficacy for diabetes management in young children. Child Health Care. 2014;43:110-119. https://doi.org/10.1080/02739615.2014. 849957.

34. Patton SR, Dolan LM, Henry R, Powers SW. Parental fear of hypoglycemia: young children treated with continuous subcutaneous insulin infusion. Pediatr Diabetes. 2007;8:362-368. https://doi.org/10.1111/ j.1399-5448.2007.00242.x.

35. Boggs SR, Eyberg S, Reynolds LA. Concurrent validity of the Eyberg child behavior inventory. J Clin Child Adolesc Psychol. 1990;19:75-78. https://doi.org/10.1207/s15374424jccp1901.

36. Cattell RB. The Scree Test for the number of factors. Multivariate Behav Res. 1966;1:245-276. https://doi.org/10.1207/s15327906mbr0102_10.

37. Beck AT, Alford BA. Depression: Causes and Treatment. 2nd ed. Philadelphia, PA: University of Pennsylvania Press; 2009. doi:https:// doi.org/10.1017/CBO9781107415324.004

38. Park CL, Helgeson VS. Introduction to the special section: growth following highly stressful life events--Current status and future directions. J Consult Clin Psychol. 2006;74:791-796. https://doi.org/10. 1037/0022-006X.74.5.791.

39. Helgeson VS, Reynolds KA, Tomich PL. A meta-analytic review of benefit finding and growth. J Consult Clin Psychol. 2006;74(5):797816. https://doi.org/10.1037/0022-006X.74.5.797.

40. Cheng ST, Lau RWL, Mak EPM, Ng NSS, Lam LCW. Benefit-finding intervention for alzheimer caregivers: conceptual framework, implementation issues, and preliminary efficacy. Gerontologist. 2014;54: 1049-1058. https://doi.org/10.1093/geront/gnu018.

41. Miller KM, Foster NC, Beck RW, et al. Current state of type 1 diabetes treatment in the U.S.: updated data from the t1d exchange clinic registry. Diabetes Care. 2015;38(6):971-978. https://doi.org/10.2337/dc15-0078.

How to cite this article: Pierce JS, Wasserman R, Enlow P, Aroian K, Lee J, Wysocki T. Benefit finding among parents of young children with type 1 diabetes. Pediatr Diabetes. 2019; 20:652-660. https://doi.org/10.1111/pedi.12860 